\title{
The Electron in Relation to Chemistry.
}

THE Faraday Society's conference on "The Electronic Theory of Valency," held at Cambridge on July $\mathrm{r}_{3}$ and I 4 , may be regarded as marking a new stage in the welding together of physics and chemistry, which has been so notable a feature of the recent history of these two sciences. The conference was attended by about 120 delegates from different universities, about half of whom were drawn from outside Cambridge. Some forty visitors were entertained in Trinity Hall, through the kindness of the master and fellows, to whom a deep debt of gratitude is due for contributing in this way to the pleasant social features of the conference. The foreign guests included Prof. G. N. Lewis, Prof. W. A. Noyes, Prof. Lyman, and Prof. Victor Henri of Zurich; the physicists included Sir J. J. Thomson, Sir Ernest Rutherford, Sir William Bragg, Prof. Barton, Prof. W. L. Bragg, Prof. Porter, Prof. Rankine, Dr. F. W. Aston, and Mr. R. H. Fowler; the chemists, in addition to Sir Robert Robertson, the president of the Faraday Society, included Sir William Pope, Prof. Heilbron, Prof. Lapworth, Prof. Lowry, Prof. Robinson, Prof. Smithells, Prof. Thorpe, Mr. C. R. Bury, Dr. Flürscheim, Dr. W. E. Garner, Dr. Henstock, Dr. Kenner, Mr. W. H. Mills, Mr. E. K. Rideal, and Dr. N."V. Sidgwick.

The conference was held in the new Department of Physical Chemistry, which is housed very appropriately in a block of buildings lying between the Chemical Laboratory in Downing Street and the Cavendish Laboratory in Free School Lane. These buildings, which were formerly in the occupation of the Department of Engineering, now provide ideal quarters for work in physical chemistry. They have been completely refitted and are admirably suited to their new use; they are also so commodious that there is a reserve of floor-space which has been loaned to workers from other departments, pending the time when it may be required for further extensions of physico-chemical work. Tea was served before the conference opened, in the large laboratory of the Hopkinson wing, which was erected in 1900 as a memorial to the late Prof. Bertram Hopkinson and his son, while the discussions were held in the lecture theatre adjoining.

The Friday afternoon session, dealing mannly with the application of the electronic theory to the problems of inorganic chemistry, was presided over by Sir J. J. Thomson, who in his opening address referred to the fact that, while the force which retains the electrons in an atom is proportional to the positive charge on the nucleus, the disruptive force which tends to make them scatter is proportional to the third power of their number, so that a limit is set to the number of electrons which can be crowded into one atom. The law of force is such that when the number of electrons is small, the octet is a particularly stable grouping; but with a different law of force, a sextet might be more stable than an octet. The problem of molecular structure can be attacked most readily by the study of cases of substitution; thus the electric moment introduced on replacing hydrogen by chlorine can be calculated, and measurements of the specific inductive capacity of molecules of different types have shown that this moment is constant in magnitude.

Prof. G. N. Lewis, in presenting his paper on Valence and the Electrons," directed attention to the reconciliation which has recently taken place between the views of physicists and chemists in reference to the structure of the atom. Since physicists have now adopted a model in three dimensions, it is possible to regard the orbit of the electron as having a fixed orientation, although the electron itself is in rapid motion. The chemist's theory of static electrons has, therefore, been merged quite naturally into a scheme of static orbits. Prof. Lewis directed attention to the fact that, in Bohr's atomic structures, each of the rare gases from neon to niton, and all the stable elementary ions, possess eight electrons in the outer shell, thus affording full justification for what came to be known as "the octet theory." The fundamental phenomenon of chemistry is, however, the formation of pairs of electrons; and of some hundred thousand known substances only about half-a-dozen contain uneven numbers of electrons. This pairing may perhaps be due to magnetic forces, since unpaired electrons always give rise to a magnetic moment. When four of these electron-pairs are grouped at the corners of a regular tetrahedron the still more stable configuration of the octet is obtained.

Prof. Lewis attaches great importance to the view that the sharing of a pair of electrons constitutes a chemical bond between two atoms. When this bond is broken, the electron-pair usually remains attached to one atom, which acquires a negative charge, while the associated atom (which loses its share of the electron-pair) acquires a positive charge on disraption. This contrast is described in Langmuir's nomenclature as the conversion of a "covalence" into an " electrovalence" ; and most English readers have accepted this nomenclature as an essential feature of the "Lewis-Langmuir hypothesis." Prof. Lewis, however, regards the ionised bond as being no longer a bond at all, and even objects to the use of the term "valence" to express the electrical state of the atom, although for nearly seventy years bismuth and aluminium have been described, like phosphorus and nitrogen, as tervalent elements.

The two following papers; by Mr. R. H. Fowler on "Bohr's Atom in Reference to the. Problem of Covalency" and by Dr. N. V. Sidgwick on "The Nature of the Non-polar Link," were of interest as exhibiting two parallel lines of thought in the application of Bohr's theory of the structure of atoms to the unsolved problem of the electronic structure of molecules. The close agreement between the conclusions reached on this subject at Oxford and at Cambridge is noteworthy. As might perhaps have been anticipated, the Cambridge physicist was much more apologetic than the Oxford chemist, since he evidently realised more fully the risks that must be taken when forsaking the mathematical concepts, verified by observations of spectra, on which the structure of the atom is based, for purely qualitative conceptions of molecular structure, which are at present beyond the range of mathematical analyses and of experimental verification. The chemist, on the other hand, boldly translating the shared electrons of Lewis into shared orbits (compare Dr. N. P. Campbell's letter in NATuRE of April 28, p. 569), was ready at once to gather a harvest of new conceptions from this speculative extension of Bohr's theory. A study of the printed papers shows, however, no important discrepancies between the views of the two authors as to the results of extending the theory of orbits from atomic to molecular structure.

In the discussion following upon the reading of these papers, Sir J. J. Thomson pointed out that two electrons are not necessary to make a bond, since $\mathrm{H}_{2}{ }^{+}$is one of the most persistent aggregates met 
with in positive rays, although there is only one electron left to hold the two protons in combination. To this Mr. Fowler replied that although this may be stable for an indefinite period in a vacuum, aggregates of this type appear to be quite incapable of resisting chemical combination-perhaps because an odd electron passing from one nucleus to the other would impart an electrical charge alternately to one atom and the other, giving rise to an unstable condition which would be particularly ready to undergo chemical change.

Prof. W. A. Noyes, after reviewing briefly the history of the development of the theory of ionisation, laid stress on the fact that the distinction between polar and non-polar union is one of degree and not of kind, the fundamental factor in both types of union being the pairing of electrons. He also directed attention to the fact that the elements, such as lithium, sodium, potassium, rubidium, and cæsium, which are monoatomic in the gaseous condition, are exactly those which have a single valency-electron in the outer shell.

Sir William Bragg made an important statement in reference to the lengths of the carbon chains in the fatty acids and esters. Each additional carbon atom in the alcohol radical increases the length of the carbon chain by $\mathrm{I}_{2} 22 \AA$.U.; but for the acid radical the average increment is only $0.97 \AA$.U. This remarkable result can be explained, while maintaining a fixed distance of $I \cdot 5 \AA$.U. between the carbon atoms, by assuming, on the alcohol side of the molecule, a simple zigzagging of the chain of atoms, with a fixed tetrahedral angle of $109^{\circ} 28^{\prime}$, the branching of the chain being to the left and right alternately. The increment on the acid side of the chain can be explained by assuming the formation of a zigzag chain of another type, the deflexions being in the order LLRRLL, etc., instead of LRLRLR. These two forms of zigzag appear to be initiated by the two types of oxygen-linking in the $-\mathrm{CO} . \mathrm{O}-$ group, and then to be rigidly maintained in the two chains. The structure suggested on the acid.side of the molecule may explain the alternation of physical properties observed in the well-known odd and even series of acids, since the increment of length is alternately parallel to the chain and inclined at an angle of $109^{\circ} 28^{\prime}$ to it.

At the close of the session Prof. Victor Henri made a brief communication in anticipation of the important paper which he delivered on the following day. The discussion took place under tropical conditions, which were so extreme that the session was adjourned before the discussion had become completely informal. An informal discussion was, however, carried on in the cooler atmosphere of the evening in the fellows' garden of Trinity Hall, to which some sixty delegates adjourned after dining together in the Hall of the College. The bringing into direct personal contact of workers who had previously known one another only by correspondence, or by reading one another's published communications, was a most valuable feature of the conference, and full advantage was taken of the opportunities thus presented.

The discussion, on Saturday morning, of the applications of the electronic theory of valency to organic chemistry, was presided over by Sir Robert Robertson. In opening the discussion, Prof. Lowry urged that the electron has come to stay, and that sooner or later organic chemists must take into consideration the electronic structure of atoms and molecules. These may prove to be a mere translation into a new language of the structural formulæ of Kekulé and van 't Hoff; giving rise to a new nomenclature but to no new conceptions. This is, however, unlikely in view of the enormous advances that have followed from the discovery of Dalton's atom, and of each fresh detail of its structure. The electronic theory of valence has already made a contribution of real value by discriminating between two types of valency, since a single bond can now be classified as depending either upon electron-sharing or upon electrontransference. Prof. Lowry's own contribution had consisted in the suggestion that a double bond may assume a form in which one linkage of each type is present. This has led to a number of novel conclusions which have been set out in a paper published in the April number of the Journal of the Chemical Society and in a paper on "Intramolecular Ionisation in Organic Compounds" contributed to the present discussion.

In summarising a second paper on "The Transmission of Chemical Affinity by Single Bonds," Prof. Lowry raised the question as to how many types of valency the chemist would wish the physicist to provide, and how many different mechanisms must be invented to account for the transmission of chemical affinities through chains of atoms. Prof. Lowry believes that only two types of valency are necessary, and that principal and subsidiary valencies, partial valencies, conjugated double bonds, carbonium bonds, mobile hydrogen atoms, centric bonds and paralinkages in aromatic compounds, are all manifestations of those electrostatic forces which Langmuir describes as electrovalence. In the same way, the unidirectional "general " effect, and the "alternating" effects observed in conjugated chains, appear to account for nearly all the phenomena observed in the transmission of chemical affinity. Lapworth and Flürscheim have suggested cases in which alternating effects appear to be produced in chains of single atoms; but other explanations (such as the steric effects described by Sir William Bragg) appear to be capable of accounting for most of these observations, and further evidence is needed before a third mechanism of transmission need be admitted. The evidence now brought forward by Lapworth and Robinson may perhaps provide the unexplained residue of observation which would make such a mechanism necessary. Prof. Lowry's paper also contained a vindication of Vorländer's view that, when its direct neutralising action is eliminated, the amino-group possesses well-defined acylous properties; it is therefore no anomaly for an aminoacid to be stronger than the fatty acid from which it is derived.

Prof. Lapworth, in communicating a paper on "Some Recent Contributions to the Theory of Induced Alternate Polarities in a Chain of Atoms," described six different theories which have been put forward in order to account for these phenomena. In criticism of the previous speaker, he stated that he himself twenty years previously emphasised the tendency of organic compounds to assume a "homogeneous " in place of a " heterogeneous " distribution of valency. This is precisely the same phenomenon that Prof. Lowry discussed under the heading of "Crossed Polarities." As evidence of alternate polarities in chains of atoms held together by single bonds he quoted the biochemical oxidation of butyric acid to $\beta$-hydroxybutyric acid and then to acetoacetic acid. This case has also been quoted independently by Robinson.

Prof. Robinson contributed a paper on "Octet Stability in Relation to Orientation and Reactivity in Carbon Compounds." He directed attention, as Prof. Lapworth had done, to the fact that optical activity is often preserved in chemical changes,

NO. 280.5 , VOL. I I 27 
although, if the intermediate stages are those which are conventionally postulated, the optical activity must necessarily disappear, since the intermediate product would be symmetrical. The preservation of asymmetry can, however, be explained by means of partial valencies, through which the asymmetry of one atom is maintained until it has been stabilised again either in the same atom or in a different one.

The bearing of the theory of polarity on the reactivity of organic compounds was discussed by $\mathrm{Mr}$. E. K. Rideal, and, as illustrating this aspect of the problem, Mr. R. G. W. Norrish described some experiments which he has just carried out, according to which the union of ethylene and bromine is almost stopped by enclosing the mixed gases in a vessel lined with paraffin wax, whereas in contact with the polar surface of a glass vessel combination takes place rapidly.

In the general discussion, to which about a dozen different speakers contributed, Prof. J. F. Thorpe urged that the theory of polarity " explains everything but predicts nothing," in marked contrast to van 't Hoff's stereochemistry, which made organic chemistry into the most exact of all the sciences. This contention was strenuously denied by $\mathrm{Mr}$. Burkhardt from the Manchester laboratory and by Profs. Noyes, Lapworth, Heilbron, and Robinson, who proceeded to put on record two definite predictions in order to get over the difficulty that under normal conditions the prediction and the verification are published together, so that the reader cannot be quite certain which really came first. Dr. Flürscheim also replied in a very vigorous manner to the criticisms by Prof. Lowry of his views in reference to the influence of substitution on the strength of carboxylic acids, and urged that in several examples the formation of internal salts, which had been suggested as an alternative explanation by Prof. Lowry, cannot in fact take place. Prof. Lewis directed attention to the fact that the breaking of a double bond does not necessarily get rid of cis and trans isomerism, even if free rotation can take place; and in support of this view Mr. Bury quoted the fact that quadrivalent sulphur compounds retain their optical activity even when one of the four groups is ionised.

In the final session of the conference, Prof. Victor Henri presented a paper on "Molecular Polarity, deduced from the Study of Absorption Spectra." This proved to be a most remarkable contribution, in which the application of considerations based on the quantum theory led to the conclusion that quantified motion may occur in electrons, atoms, or molecules, giving rise to broad absorption-bands, narrow absorption-bands, and a fine structure of these bands, respectively. By making use of a source of continuous ultra-violet light, Prof. Henri has been able to study the fine structure of the absorption bands of a large number of compounds, and in some instances to measure as many as two thousand bands in the fine structure. A large number of photographs were shown to illustrate the various types of absorption spectra. Prof. Henri's paper produced a very profound impression by the masterly character both of the theory and of the experiments which he described. It may well mark a new era in the history of absorption spectra. In apologising to Prof. Henri for the fact that the late hour did not allow of an opportunity for discussion, the president added that the question of holding a general discussion on absorption spectra is already under consideration by the council of the Faraday Society, and that such a discussion would allow of a fuller consideration of the view which Prof. Henri had put forward.

\section{International Conference of Phytopathology and Economic Entomology.}

THE first International Conference of Phytopathology and Economic Entomology was held in Holland on June 24-July 2 by the kind invitation of the Netherlands Government in co-operation with the authorities of the various laboratories and institutes conducting work on agricultural phytopathology and entomology. The Conference was noteworthy as being the first occasion on which phytopathologists and entomologists from all countries have been invited to meet together to discuss matters of mutual interest. Prof. H. M. Quanjer of Wageningen, presided over the Conference, while Dr. L. O. Howard, Chief of the United States Bureau of Entomology, acted as president of honour. Upwards of 65 members attended-in addition to the Dutch participantsrepresenting some 26 countries. A report of the Conference, giving an account of the demonstrations, papers read, etc., will be issued by the Committee of Management (obtainable from the Secretary, Mr. T. A. C. Schoevers, of the Netherlands Phytopathological Service, Wageningen), but in the meantime the following brief account may be of interest.

Members assembled at Wageningen on June 24, when an address of welcome was delivered by Prof. Kielstra, Rector Magnificus of the University. On the following morning the Conference was formally opened by H.E. the Minister for Home Affairs and Agriculture, and during the day members attended the inauguration, by Jonkheer van Citters, of the new Laboratory for Potato Research in which Prof.
Quanjer's Department is now housed. In the laboratory and adjoining experiment station experiments of great interest, notably in connexion with the "virus" diseases of the potato, were inspected. After spending two days in conference, the members divided into two parties, one visiting Groningen and the other Boskoop, Aalsmeer and Haarlem. The first party inspected the practical application of Prof. Quanjer's experiments in the selection fields and farm of Dr. O. Botjes, who demonstrated his methods of obtaining "seed" potatoes on a large scale entirely free from virus diseases, and the second visited nurseries producing ornamental plants, shrubs and bulbs; at Lisse (near Haarlem) they visited Dr. van Slogteren's new laboratory for the study of bulb diseases, and were treated to an excellent address and to demonstrations. The two parties combined forces again at the laboratory of Prof. Westerdijk, Director of the W. C. Scholten Phytopathological Laboratory at Baarn, where the final papers were read and the discussions concluded. Subsequently H.E. the Minister for Home Affairs and Agriculture held a farewell reception at the Hague, and members were entertained to dinner at Scheveningen. Many members remained until the following week to take part in an expedition to the glass house district of the Westland.

Within a short space it is impossible to do justice to the papers read. Two subjects were, however, specially prominent-namely, (I) the research, both botanical and entomological, which centres round the

$$
\text { NO. } 2805 \text {, VOL. I } 127
$$

